Paediatr Paedolog 2018 · 53:76-79

https://doi.org/10.1007/s00608-018-0556-7

Online publiziert: 26. Februar 2018

(c) Der/die Autor(en) 2018. Dieser Artikel ist

eine Open-Access-Publikation.

CrossMark

P. Scheer

Graz, Österreich

\title{
Zu viel Stress?
}

\section{Anleitung zu einem neuen Umgang für Kinder- und Jugendfachärzte}

\section{Alle Lebewesen haben Stress}

Hans Seyle hat aus dem Stressphänomen das Beste herausgeholt. Er zeigte in seinen Publikationen, dass alle Lebewesen Stress haben und empfinden. Vom Pantoffeltierchen, das in einer hyperosmolaren Lösung Stress empfindet und fliehen will, bis zum Homo sapiens sapiens - alle kommen in Stresssituationen und versuchen ihnen durch Flucht, oder in der späteren Phylogenetik, durch Kampf $\mathrm{zu}$ entgehen. Stress ist ein durch äußere Einflüsse ausgelöstes Phänomen, bei dem durch eine akute Ausschüttung von Adrenalin Reaktionen ausgelöst werden, wie sie für Flucht oder Kampf erforderlich sind. Die Umwandlung des Worts Stress als ein Synonym für Belastung, Überbelastung oder gar schädliche Belastung hat dieser Definition keinen guten Dienst erwiesen [1]. Denn dadurch kann das an sich erforderliche physiologische Phänomen, das für Vigilanz und Wachsamkeit nötig ist, nicht mehr als solches beschrieben werden. Der Bedeutungswandel des Worts hat es in eine Richtung bewertet.

\section{Stress in der Ordination}

In der kinder- und jugendärztlichen Praxis unterscheiden wir aus didaktischen Gründen folgende Stressauslöser:

1. mangelnde Vorbereitung/ Überschätzung,

2. mangelnde Übung und Evaluierung, 3. mangelnde Copingstrategien oder

4. fehlende Pausen bzw. fehlende Entspannung bzw. mangelndes Lob.

\section{Mangelnde Vorbereitung}

Jede kinder- und jugendärztliche Praxis benötigt Vorbereitung. Entweder im Terminmanagement oder in der Art, wie Patienten zugewiesen oder abgeschlossen werden. Heute werden die meisten dieser Aufgaben an einen Computer delegiert. Daher bleiben wir bei dem, was nicht delegiert werden kann; das umfasst:

1. Einsicht in die eigenen Kenntnisse und Möglichkeiten,

2. Einsicht in die Kenntnisse der Mitarbeiter und

3. Unvorhergesehenes.

Die Fehleinschätzung bezüglich der eigenen Kenntnisse und Möglichkeiten gehört zu den entscheidendsten Stressfaktoren. Weder kann man in einer ultrakurzen Zeit einem Patienten und seiner Familie gerecht werden - auch wenn das manchmal als erforderlich erscheint, noch kann man alles können, noch kann man zu allen Zeiten gleich gut sein. Das alles einzusehen, bedarf der Selbsterkenntnis. Wie ich in einer Fortbildung der Steirischen Fachärztegruppe erwähnt habe, glauben alle Ärzte, dass sie alles können: selbst das, was sie lange nicht geübt haben oder nie wirklich beherrschten. Diese Annahme führt im Anspruchsfall zu großem Stress. Es mag sogar sein, dass sie im Hintergrund zu Stress führt, weil sich der Arzt kein Sicherheitsnetz baut, auf das er im Bedarfsfall zurückgreifen kann. Wir sind nicht in Schwarzafrika des vorigen Jahrhunderts und können und müssen daher nicht entbinden und appendektomieren können. Wir müssen in unserem Rahmen bleiben, wenn wir auch bisweilen etwas darüber hinaus- 
gehen sollen und müssen, um unsere Grenzen wahrzunehmen.

Die Mitarbeiter sind ebenfalls kritisch einzuschätzen. Es gibt in der Tat Menschen, denen eine selbstständige Entscheidung Angst macht. Sie neigen dazu, den Arzt mit jeder Kleinigkeit zu belasten. Andere wieder sind zu selbstständig. Eine gute Mitarbeiterführung und -anleitung ist erforderlich, wenn man nicht ununterbrochen gestört werden will, was dann in Stress ausarten kann.

Der Platz für Unvorhergesehenes ist unabdingbar. Im Grunde ist jeder Arzt ein Troubleshooter. Alle anderen Selbsteinschätzungen weise ich zurück. Daher sollten Pausen in ein Bestellsystem eingetragen werden, in denen unvorhergesehene Probleme bearbeitet werden können. Schaut man auf Terminkalender von niedergelassenen Kollegen, so findet man selten Pausen als solche eingetragen. Ich werde später noch über deren Wichtigkeit sprechen, jetzt nur so viel: Sie sind als Puffer erforderlich.

\section{Mangelnde Übung und Evaluierung}

Alles was man kann, kann man, weil man es gelernt und danach immer wieder geübt hat: Sprache, Fitness und vieles mehr. Ebenso geht es in der Praxis: Hat man sich entschlossen, Blut- oder Ultraschalluntersuchungen zum unverzichtbaren Inventar der Praxis zu machen, dann übt man es. Das ist jedem klar. Hat man aber auch andere Ideen, wie z. B. im Notfall Akuthilfe anbieten zu können und $\mathrm{zu}$ müssen, dann soll man das üben. Am besten mit allen Mitarbeitern der Praxis; je überraschender und realitätsnäher, desto besser. Was man nicht übt, sollte man auch nicht machen (wollen), weder Reanimation über den Beitel hinaus noch Lumbalpunktion. Sogar Leitungen bei einem Neugeborenen zu legen, muss immer wieder geübt werden. Ich weiß schon, dass wir uns erinnern. Wir erinnern uns an unsere Heldentaten. Aber haben wir in der Praxis die Ausrüstung, die Schwester, die uns das Kind hält, und die Geduld, mehrere Male zu stechen? Und wenn wir dann den Venenzugang haben: Was machen wir damit? Geben

Paediatr Paedolog 2018 · 53:76-79 https://doi.org/10.1007/s00608-018-0556-7

(c) Der/die Autor(en) 2018. Dieser Artikel ist eine Open-Access-Publikation.

\section{P. Scheer}

\section{Zu viel Stress? Anleitung zu einem neuen Umgang für Kinder- und Jugendfachärzte}

\section{Zusammenfassung}

Keine Frage, die Ordinationen von Kinderund Jugendfachärzten sind Hotspots. Kranke Kinder warten neben gesunden. Mütter tauschen sich über ihre Kinder und oft auch über den Kinderarzt aus. Da Krankheit immer ungelegen kommt und Kinder sich manchmal vor dem zu Erwartenden fürchten, ist weder der Patient noch sein Angehöriger gerne dort. Der vorliegende Beitrag schlägt Ideen und Maßnahmen vor, wie die Situation verbessert werden kann. Dabei wird auf Stressvermeidungskonzepte aus dem Management zurückgegriffen. Dort wie da scheinen Änderungen schwierig. Zu sehr scheint die äußere Situation und die gestellte Aufgabe die Anspannung zu definieren.
In manchen Branchen scheint es immer schnell gehen zu müssen. Zeit für Überlegung und Planung scheint zu fehlen. Doch nur Unternehmen mit einer lernenden Kultur, die imstande sind, sich an neue Gegebenheiten anzupassen, können langfristig überleben. So geht es auch dem Kinder- und Jugendarzt in der Ordination: Vorausschauende Planung und der Einsatz von Copingstrategien sowie das Wissen um Resilienz als Wundheilung helfen, Patienten langfristig kompetent und vor allem freudig versorgen zu können.

Schlüsselwörter

Stress · Planung $\cdot$ Coping $\cdot$ Evaluierung .

Pausen

\section{Too Much Stress. Guidance on a New Approach for Pediatricians}

\section{Abstract}

There is no question that pediatricians' surgeries are hot spots. Sick children wait next to healthy ones. Mothers exchange views on their children and often their pediatrician too. Because illness always comes at an inconvenient time and the children are sometimes afraid of what to expect, neither the patient nor their relative wants to be there.

The present article suggests ideas and measures on how this situation can be improved. It refers to stress avoidance concepts from business management. There, as here, it can be difficult to make changes. Both the external situation and the set task seem to be defined by too much tension. In some sectors, everything has to happen fast. Time to ponder and plan seems to be lacking. However, only businesses with a culture of education that are capable of adjusting to new circumstances can survive in the long term. This is the case with pediatricians in the surgery too: proactive planning and applying coping strategies, as well as awareness regarding resilience as wound healing, eventually help doctors to care for patients competently and above all with pleasure.

\section{Keywords}

Stress · Planning · Coping · Evaluation · Breaks wir eine häusliche Infusion? All das will überlegt und vorsorglich all jenes weggeräumt sein, das nicht mehr gebraucht wird. Sonst haben wir es, denken daran und im Anlassfall macht es Stress und ermöglicht vermeidbare Fehler.

Im Fehlermanagement gilt es darauf zu achten, dass Fehler zugegeben werden können. Zumeist werden aus Missgeschicken und echten Fehlern erst wirkliche, lebensbeeinträchtigende Fehler (sei es aufseiten des Patienten mit einer fortdauernden Gesundheitsstörung oder aufseiten des Arztes mit einer fortdauernden Gerichtsproblematik), wenn sie nicht besprochen werden. Wird der Fehler gleich und unmittelbar eingestanden, kann an der Schadensbegrenzung gearbeitet werden. Daher:

1. Überfordern sie weder sich noch andere.

2. Üben sie das Notwendige. Geben sie das Überflüssige weg.

3. Trachten sie danach, eine strafarme Kommunikation zu haben. Machen sie klar, dass das Verschweigen schlimmer ist als das Geständnis. ${ }^{1}$

${ }^{1}$ Lesen Sie dazu Stefan Zweig: Angst. 


\section{Mangelnde Copingstrategien}

Mit Coping bezeichnet man heute die Fähigkeit, Schwierigkeiten zu überwinden, anstehende Probleme zu meistern und bestenfalls zu lösen. Gelingt das Coping nach einem stressenden Erlebnis, spricht man von Resilienz ${ }^{2}$, wenn die ursprüngliche psychische Verfassung wieder hergestellt werden kann. Copingstrategien sind individuell erlernt, meist laufen sie unbewusst $\mathrm{ab}$ und sie sind von Mensch zu Mensch verschieden. Manche verschließen sich, manche reden viel, manche lösen einfach das Problem und wieder andere rennen weg. Sie sind Erwachsenen oft bekannt. Viele denken, dass sie unveränderbar sind.

Unter Organisationskultur werden unter anderem auch jene Copingstrategien zusammengefasst, die in einem Unternehmen oder einer ökonomisch definierten Gruppe vorherrschend sind. Es ist anzuraten die Strategien zu verwenden, die in dem jeweiligen Unternehmen üblich sind. Bisweilen sind diese Strategien nicht hilfreich, aber so vertraut, dass eine Änderung als Verstoß gegen die Usancen erlebt wird, was auch als Tabubruch bezeichnet werden kann. In ärztlichen Unternehmen sind solche Tabubrüche gut. Sie dienen dazu, eingefahrene Fehler oder falsche Abläufe zu korrigieren.

Man sollte daran denken, dass der Mensch unter Stress um ein Vielfaches fehleranfälliger ist. Wir machen im Stress mehr Fehler, weil unser Fokus vor allem auf Kampf oder Flucht liegt und wir alles andere als Behinderung erleben. Daher besteht die Idee, dass jede Meldung zugelassen werden soll. Amerikanische Filme leben oft davon, dass z. B. einem Kind nicht zugehört wird. Dieses Kind hat aber die entscheidende Beobachtung gemacht, die entweder den Mörder überführt oder sogar die Welt rettet.

Denken sie also daran: Copingstrategien, die dysfunktional geworden sind,

\footnotetext{
2 Der Ausdruck Resilienz kommt aus der Erzeugung von pneumatischen Reifen. Diese wurden darauf getestet, ob sie nach einem Eindruck (im Wortsinn also z.B. durch einen Stein) wieder die runde, ursprüngliche Form annehmen konnten.
}

müssen geändert werden. Wenn in der Grippezeit jeden Tag die Mannschaft der Ordination am Abend gerädert ist, dann sind weder Grippezeit, noch lästige Eltern, noch das unnötige Aufsuchen der Ordination schuld, sondern ihre Organisation kann das nicht bearbeiten, wozu sie gewidmet ist: die Versorgung kranker Kinder. Ich weiß, ich habe das schon oft gesagt. Man hat mir geantwortet, dass ich keine Ahnung habe. Das nehme ich zur Kenntnis. Ich weiß aber, dass es nicht sein kann, dass Kinder- und Jugendfachärzte jedes Jahr von November bis Februar überlastet sind. Da läuft etwas falsch, und ich lade Sie, liebe Leser, gern ein, mit mir darüber nachzudenken.

\section{Fehlende Pausen}

Nun zum letzten Punkt meiner Ausführungen: Die Pausen! Es gibt zumeist keine Pausenplanung. Pausen entstehen aber nicht von selbst.

In einem meiner Texte zu Stressmanagement [2] habe ich als Beispiel einen Dirigenten genommen, der sich wochenlang vorbereitet, dann die Aufführung hat, Applaus und dann Ruhe. Ich weiß nichts ist so, wie es scheint. Der Kinderarzt ist ein Versorger, kein Dirigent. Was ich aber auch weiß ist: Pausen und erlebter Erfolg nützen. Sie reduzieren den akuten Stress und machen neue Anspannung möglich.

Hier sehe ich großen Handlungsbedarf bei uns, im Sinn der Versorgung der Patienten in Ruhe und Sicherheit, aber auch im Sinn eines gesunden Lebens für den Arzt selbst.

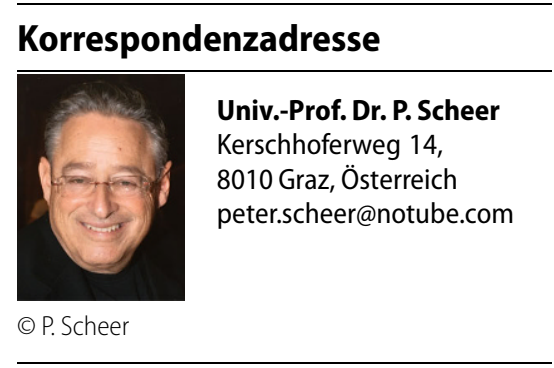

Funding. Open access funding provided by Medical University of Graz.

\section{Einhaltung ethischer Richtlinien}

Interessenkonflikt. P. Scheer gibt an, dass kein Interessenkonflikt besteht.

Dieser Beitrag beinhaltet keine von den Autoren durchgeführten Studien an Menschen oder Tieren.

Open Access Dieser Artikel wird unter der Creative Commons Namensnennung 4.0 International Lizenz (http://creativecommons.org/licenses/by/4.0/deed. de) veröffentlicht, welche die Nutzung, Vervielfältigung, Bearbeitung, Verbreitung und Wiedergabe in jeglichem Medium und Format erlaubt, sofern Sie den/die ursprünglichen Autor(en) und die Quelle ordnungsgemäßnennen, einen Linkzur Creative Commons Lizenz beifügen und angeben, ob Änderungen vorgenommen wurden.

\section{Literatur}

1. Scheer P (1986) Stress. Ansporn oder Krankheit? Manage Forum 3(1):38-44

2. Scheer P, Scheer A (2012) Das on-off Phänomen. Stressbekämpfung durch Schonschaltung. In: Kasper H (Hrsg) Strategien realisieren - Organisationen mobilisieren. Linde, Wien, S127-138 
Hier steht eine Anzeige.

\section{曾 Springer}

